\title{
Data sharing of shape model with VRML environment
}

\author{
K. G. Kobayashi, K. Ujihara*, M. Hayamizu*, and M. Fujii* \\ Japan Society for Precision Engineering
}

* Toyama Prefectural University, Dept. of Mech. Systems Eng. Kosugi, Toyama 939-0398, JAPAN

Phone: +81-766-56-7500, Fax: +81-766-56-6131

E-mail: $\{$ kobayasi,ujihara,miyu\}@pu-toyama.ac.jp

Gaio Technology Co. Ltd.

3-35-1, Tsuruya, Yokohama 221-0835, JAPAN. Phone: +81-45-316-5901

\begin{abstract}
This paper proposes a data sharing method of B-rep shape models between different CAD systems via the Internet. Extensions to CAD systems and VRML browsers enable to provide quick viewing of a shape and to transfer a shape data including curved surfaces. An experimental system is developed and its results are shown.
\end{abstract}

Keywords

Shape model, data sharing, STEP, the Internet, WWW, VRML

\section{INTRODUCTION}

Increasing requirements for data sharing of product model are promoting activities in broader range of industry to develop the international standard, called as STEP (Standard for the Exchange of Product Model Data, ISO 10303 series) (Fowler, 1995). Because STEP data structure is designed to cover an overall concept of product model, STEP data provides a precise and extensive definition of the shape (STEP Part 42, 1994). Besides, static data format provided by STEP (STEP Part $21,1994)$ is not appropriate for dynamic exchanging during design process. Thus, an exchange system based on STEP may not be implemented easily and may have a relatively heavy performance based on the current information processing technology (Choi, 1997). DMAC (Design \& Modeling Applications Council) 
(DMAC, 1998) is another possible solution to share model data via the Internet. DMAC is an extension of OLE to deliver direct access to a shape model via functional interfaces. This supports referencing the shape, but modeling capability is not yet supported.

On the other hand, if a shape of product is only required for a quick presentation via the Internet, a light and easy access method is required. This demand should not be ignored because of the world-widely distributed manufacturing based on different modeling systems. For this purpose, WWW (World Wide Web) (Krol, 1992) with VRML (Virtual Reality Modeling Language) (VRML, 1996) environment is thought to be one of the best solutions. However, the VRML data is limited to simple primitives and polygonal facets and lacks topological information and also geometric accuracy of the original shape. Thus, VRML model data is limited for viewing only and is inappropriate for modeling across the Internet.

There are two conflicting requirements; a data exchange of precise shape for modeling, and a quick presentation of approximated shape. In order to satisfy both of them, we propose a data sharing method of shape model based on VRML data. For the 3 dimensional viewing, we assume a WWW browser with VRML extension, which is commonly used. For the shape data sharing, a technique is developed to reconstruct topology and geometry with curved surfaces from polygonally subdivided facets. In this paper, this concept and its implementation method are described, examples are shown, and extensions and problems are discussed.

\section{CONCEPT OF DATA SHARING}

The data sharing is achieved by three parts; sender, viewer, and receiver. Figure 1 shows the process of data transfer from one CAD system (A: sender) to a different CAD system (B: receiver), each of which handles B-rep solid models. The reverse date flow, from $B$ to $A$, is as same with this.

The sender subdivides an original B-rep model, generates VRML data as a set of triangles, and provides data via the Internet to be displayed by the viewer. Here, the viewer is assumed to be a usual VRML browser, which parses VRML version 1.0 data (scene description language) and provides 3D walk-through capability in the modeling space. For the purpose of quick viewing, it is enough to have such a non-specialized VRML environment.

The receiver includes a functionality of the viewer. It analyzes the VRML data to reconstruct a B-rep model data. If VRML data is composed of triangles and satisfies 2-manifoldness, it is not difficult to build up a B-rep triangular mesh model, which is not appropriate for modifying and designing the shape. The receiver combines a set of triangles as a face and tries to restore the original topology and geometry. Dedicated researches are done to generate a model with 
curved surfaces from a triangular mesh model (Hoppe, 1992, etc.). However, our approach does not take such a general method, but utilizes the original B-rep topological structure to perform a light response. This approach uses overlap techniques with our preceding research (Kobayashi, 1997).

The model is transformed as shown in Figure 2. (a) and (e) are the original and the result B-rep model, respectively. Faces in the original model may include curved surfaces. (b) is the intermediate status of face subdivision. (c) is a completely triangulated model, which is interpreted into VRML data with a view of (d). From the data (c), the model (e) with curved surfaces is reconstructed.

Our data sharing concept does not assure the perfectly precise shape data to be transferred. It enables a user to get a quick view with VRML environment, and, if he/she desires, to get an approximated B-rep model data. The basic technique is described in the next section.

\section{VRML DATA FOR RECONSTRUCTION}

Here, a method to transfer a topological structure is given. For a compatibility of VRML data, no extension to VRML should be taken. Geometric data is also considered, but certain restrictions are imposed.

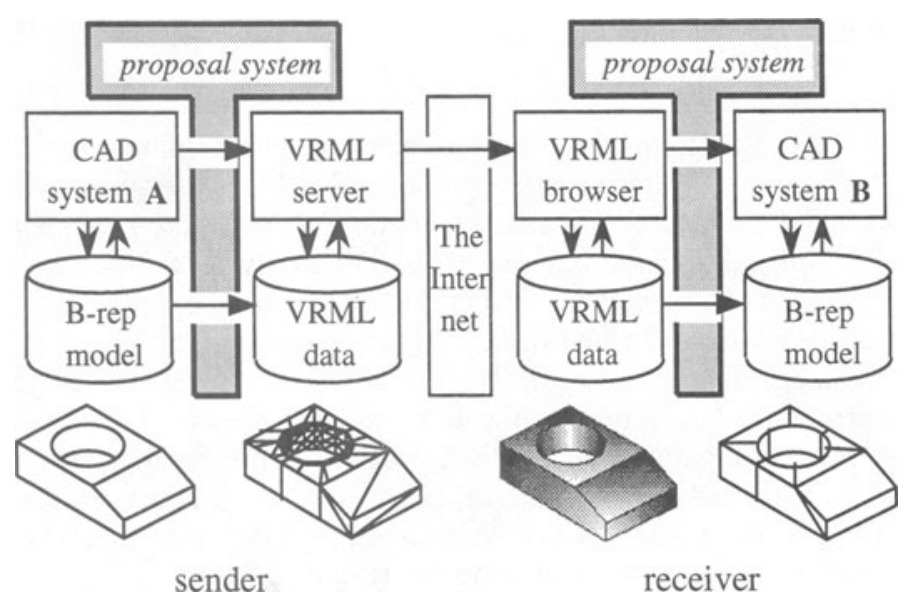

Figure 1 Concept of data sharing via the Internet. 


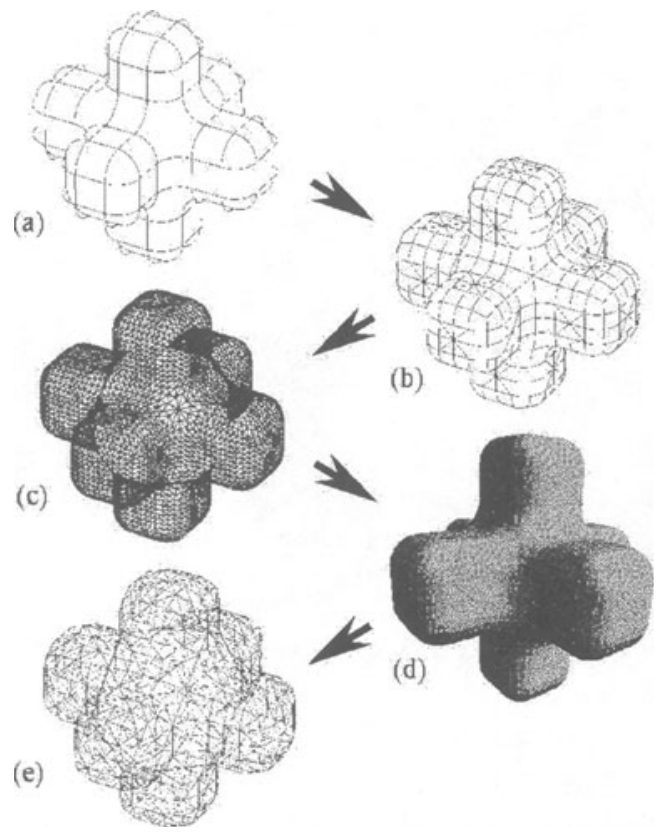

Figure 2 Models in stages of the data sharing; (a) original B-rep model, (b) subdivision of faces, (c) triangular mesh model, (d) view of VRML data, and (e) reconstructed B-rep model.

The sender prepares a triangular mesh model from the original B-rep model to subdivide each face. The face topology is bounded by loops composed of edges and vertices and may have wide varieties (Figure 3 (a)). If the face geometry is a planar surface, the subdivision is reduced to divide polygonal area into triangular portions. In case the face geometry is a curved surface, its topologically polygonal area is subdivided into four-sided patches (Figure 3 (b)) to put a mid point at the center of the face (Chiyokura, 1988). By this step, the original surface geometry is lost and approximated as a rectangular bi-parametric patch. Though each CAD system may have triangulation of arbitrary faces, it is not applicable to produce a hierarchical VRML data, described in later. This 're-patch' implementation depends on the sender CAD system. A square patch is divided in two triangular patches (Figure $3(\mathrm{c})$ ) to get triangular meshes (Figure $3(\mathrm{~d})$ ).

This triangulation process works hierarchically and its result VRML data can be represented to preserve this process as a structured data. Figure 4 shows a pattern of this structure based on grouping mechanism. Each stage of the triangulation corresponds to each depth of the VRML data, which is fully compatible as VRML and also carries the original topological structure. 


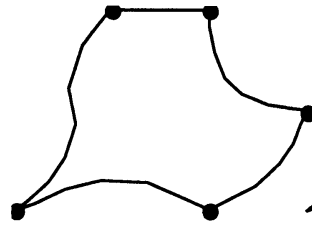

(a) face

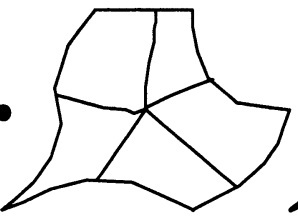

(b) square patches

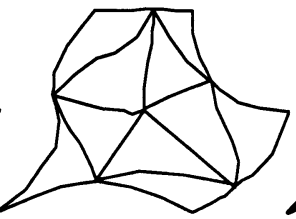

(c) triangular patches

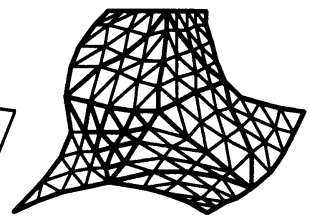

(d) triangular mesh

Figure 3 Triangulation process.

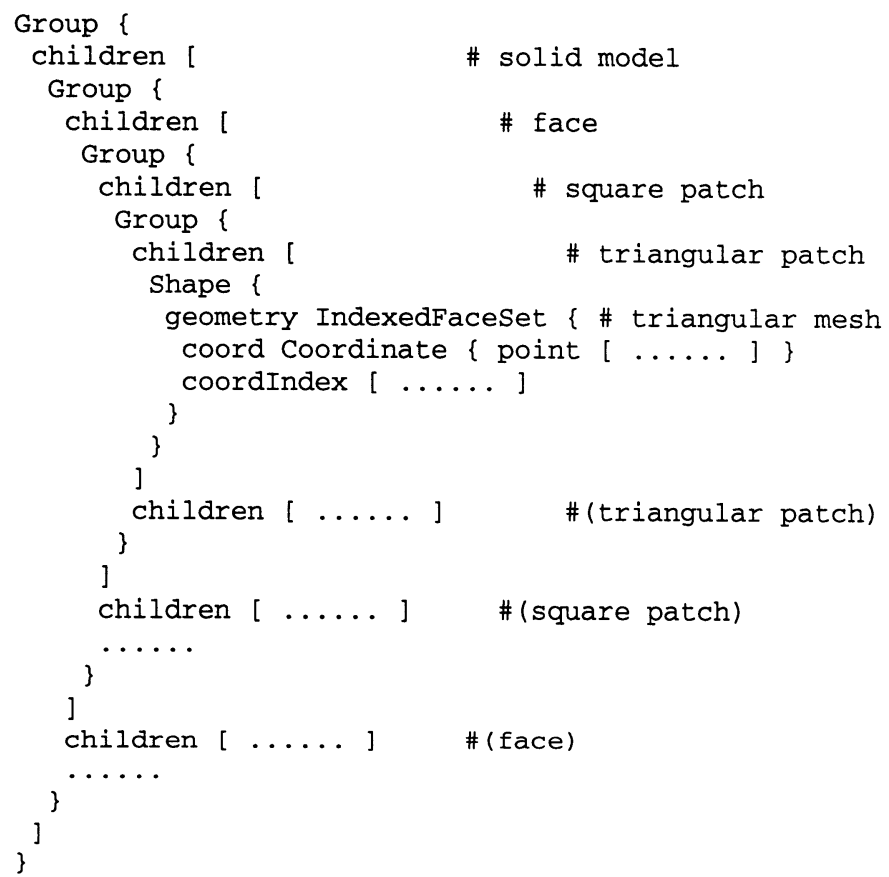

Figure 4

Hierarchical structure of VRML data.

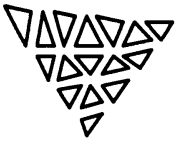

(a) triangles

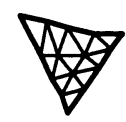

(b) triangular mesh

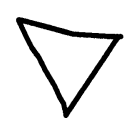

(c) face as triangular patch

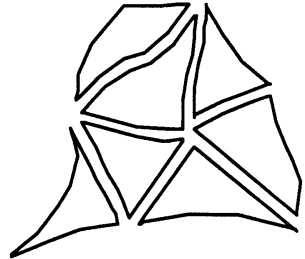

(d) face combination

Figure 5 Reconstruction process. 
The receiver analyzes the structure to recover the original topology during the reconstruction process (Figure 5). A set of triangles is elementarily grouped as a 'shape' node (Figure 4) and is interpreted as a triangular mesh (Figure 5 (b)). Because this mesh is originally generated from the half of a rectangular patch, it seems not to be difficult to rebuild a four-sided patch from a couple of triangular meshes. However, the re-patching geometry used by the sender is not necessarily covered by the receiver's geometry, and then it causes a possible discrepancy between a patch and triangular meshes. To avoid this problem, a face is primarily generated with a geometry of triangular patch (Figure 5 (c)). The curve of a boundary edge is uniquely determined by a polygon corresponding to the edge. The geometry of a triangular patch is interpolated from the boundary curves and inside points of the corresponding triangular mesh. Those triangular faces are combined as one B-rep model by using Euler operators in the receiver system (Figure 5 (d)). If faces with triangular patch geometries can be represented as one square patch or a set of smoothly connected patches by the receiver system's geometry, those faces are merged in one face. This 'patch reduction' implementation depends on the receiver CAD system.

\section{EXAMPLES AND DISCUSSIONS}

An experimental system is developed to show the effectiveness of our proposal. The target CAD modeler is DESIGNBASE version 5.1 (the Ricoh, Co. Ltd.) for both of sender and receiver. As a sender, re-patching is done by Gregory patch (Chiyokura, 1988) which is native surface geometry of the modeler. As a receiver, triangular patch is represented by Bezier triangle (Farin, 1997) for applying to general modeling systems. Here, patch reduction is not implemented due to the lack of appropriate algorithm to merge two Bezier triangles into one Gregory patch.

Figure 6 and 7 show the original models in right and the transfered models in left. Both case, overall shapes are shown to be kept. Figure 6 is a cube truncated by a free form surface on the top. The seam edge between a top face and a side face is exactly the same curve to keep geometric consistency as a B-rep model. Figure 7 is a shape like hand-dram. On the side slope, a face is reconstructed as eight triangular faces. Because continuity of smoothness between surfaces is not considered in the interpolation, there are little bumps at the most edges. This defect is also seen in the case of Figure 3 (e). The processing time of reconstruction was not enough short on SPARC 2 WS, but was reasonable comparing with the usual loading time.

The sender's major problem is system dependency. That is, re-patch to make rectangular patch before triangulation. A trimmed face, a portion of a surface arbitrarily cut by boundary curves, is the most case of re-patch. To avoid this problem, it is expected to utilize the sender CAD system's triangulation and to develop a method to compose a hierarchy of VRML data. 

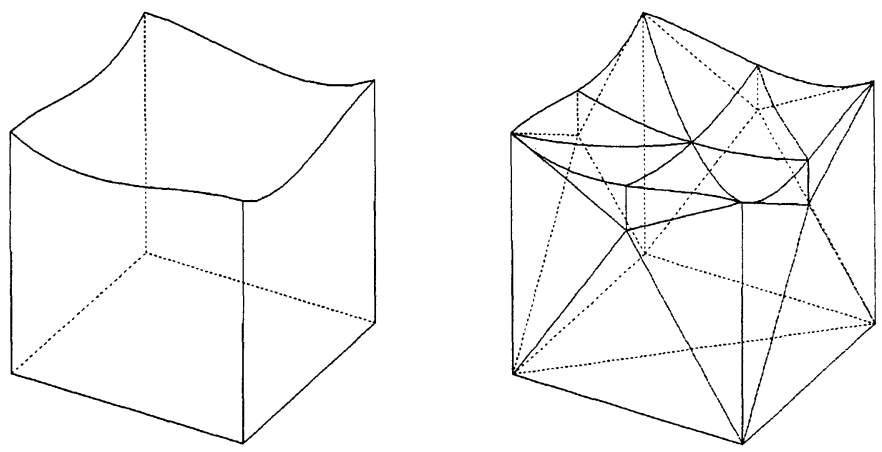

Figure 6 An example 1; left: sending model, right: received model.
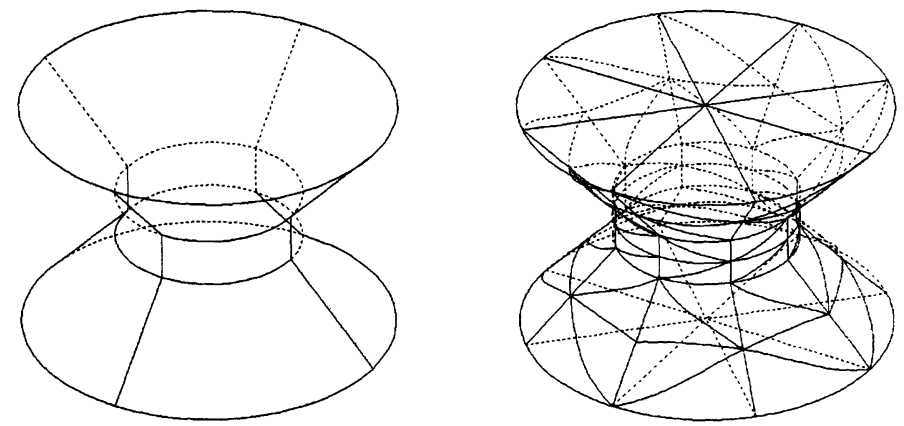

Figure 7 An example 2; left: sending model, right: received model.

The receiver's problems are incompleteness of restoring topology, and insufficient interpolation of surface geometry. It is not easy to satisfy both qualities, then the priority to topology or geometry should be specified in the reconstruction process. That is, if the geometry is mostly preferred, the topological equivalence gives way to the overall shape and smoothness. If the topology has a priority, topological elements are reduced as possible as to be equal to the original topology by ignoring $G^{l}$ continuity.

\section{CONCLUSIONS}

Our proposal is considered effective to realize two characteristics of data sharing; quick presentation and precise exchange.

Current problems and future extensions are recognized; the algorithm of surface triangulation should be reviewed with modeler independent method, and, the 
quality of reconstructed model should be controlled by priorities to geometry or topology.

\section{REFERENCES}

Chiyokura, H. (1988) Solid modeling with DESIGNBASE: theory and implementation. Addison-Wesley, U.S.A.

Choi, Y. (1997) Sharing STEP data on the Internet, in Rapid Product Development (ed. M. Ikawa, T. Kishinami \& F. Kimura), 8th ICPE, Sapporo.

DMAC (1998) Design \& Modeling Applications Council. http://www.dmac.com/

Farin (1997) Curves and surfaces for computer aided geometric design: a practical guide, 4th edition. Academic Press, U.S.A.

Fowler, J. (1995) STEP for data management, exchange and sharing. Technology Appraisals, U.K.

Hoppe, H. et al. (1992) Surface reconstruction from unorganized points. $A C M$ SIGGRAPH, 26 (2), 71-78.

Kobayashi, K. G. (1997) B-rep modeling with global modification, in Rapid Product Development (ed. M. Ikawa, T. Kishinami \& F. Kimura), 8th ICPE, Sapporo.

Krol, E. (1992) The whole Internet user's guide \& catalog. O'Reilly \& Associates, U.S.A.

STEP Part 21 (1994) IS 10303-21, Industrial automation systems and integration -Product data representation and exchange -- Part 21: Implementation methods: Clear text encoding of the exchanging structure. ISO, Geneva.

STEP Part 42 (1994) IS 10303-42, Industrial automation systems and integration -Product data representation and exchange -- Part 42: Integrated generic resources: Geometric and topological representation. ISO, Geneva.

VRML (1996), VRML Architecture Group. V.2.0 Specification. http://vag.vrml.org/

\section{BIOGRAPHY}

K. G., Kobayashi was born in Japan in 1961 and has been Assoc. Prof. of Toyama Prefectural Univ. since 1990. K., Ujihara and M., Fujii are in the graduate school of Toyama Prefectural Univ. M. Hayamizu graduated the department in 1998. We and our colleague intend to bring innovative occasions on shape modeling technology including user interface, data exchange, flexibly deforming objects, etc. 